\title{
GI-MS48-02 | Seeing is Believing: Model Finalisation ANd Interpretation of
}

\section{RESULTS}

Linden, Anthony (University of Zurich, Zürich, $\mathrm{CH}$ )

User-friendly software and tools for solving, model-building and refining structures have advanced tremendously over the last 15 years or so. Even fully-automated routines are available, which, in many cases, do a handsome job of producing an almost finished structure. This talk will look at some of the ways of going beyond the automatic and push-button procedures to ensure that the final structure model is truly correct and the best possible outcome from the data at hand. Aside from validation with checkCIF, simply looking at and rotating the structure model onscreen can give a good impression of the quality of the results; if it looks weird, it probably is. Unusual geometry, strange-looking atomic displacement ellipsoids or inexplicable residual electron density peaks can all be indicators that the structure model (or the reflection data) is deficient in some way and might be in need of additional thought. After the refinement has been completed and validated, the real objective of the study, to answer a scientific question that lead to the need for a structure determination in the first place, can hopefully be addressed. This involves interpretation of the results. The presentation will discuss how to derive, compare and correctly evaluate useful information from the structure determination, while keeping in mind the statistical significance of any numerical differences. 